\title{
Immunomodulation Effect of Nypa fruticans Palm Vinegar ${ }^{\dagger}$
}

\author{
Sa-ngob LAKLAENG and Wiyada KWANHIAN*
}

School of Allied Health Sciences, Walailak University, Nakhon Si Thammarat 80160, Thailand

("Corresponding author's e-mail: wiyadakwanhian@gmail.com)

Received: 12 April 2019, Revised: 5 July 2019, Accepted: 19 August 2019

\begin{abstract}
Vinegars are aqueous products of fermentation that are composed of acetic acid, water and other trace chemical components. Previous studies have shown that they have several beneficial effects for humans, such as anti-diabetic, anti-bacteria, antioxidant, anti-tumor or the enhancement of immune function. Nypa fruticans palm vinegar (NPV) is 1 of the vinegars that is usually used as a food ingredient. However, there are limited studies that have provided results to support its proposed immunomodulatory properties. This study investigated the immunomodulatory effects of NPV. Locally produced NPV from 3 different planting areas (fresh, brackish and saline water) of the Nakhon Si Thammarat province in Thailand were administered to rats, and the enhancement of the in vitro phagocytic activity was assessed. The results showed that NPV potently enhanced antibody production and the phagocytic activity. Moreover, NPV-treated rats showed a reduction of edema in type 4 hypersensitivity reactions. The results indicated that NPV had immunomodulatory effects, and therefore, NPV could be considered a functional food.
\end{abstract}

Keywords: Nypa fruticans, Nipa palm vinegar, NPV, Immunomodulatory effect, Functional food

\section{Introduction}

Vinegar is normally used in a variety of food ingredients. Several kinds of fruits or grains are used as the primary starting materials for vinegar production [1]. The natural vinegar product, aside from being used as part of food ingredients, has long been considered. Recently, the potential health benefits of vinegar varieties due to its functional properties and bioactive substances have been reported by many studies [2-4].

Nypa fruticans palm is abundant and found along the coasts and rivers from Bangladesh to the Pacific islands. It can grow in soft mud-like mangrove areas with fresh, brackish and saline water. Most tapped palm trees give a of nipa sap very rich in sugar $10 \%$ to $20 \%$ according to species and individual variation. In Asia, the sap is used either as fresh juice or processed into many product types, e.g. wine, alcohol, araq, sugar and vinegar, etc. [5]. Nipa sap contains sucrose, protein, and minerals, including calcium, phosphorous, iron, copper and vitamins [6]. Moreover, there contain several non-essential amino acids, glycine, glutamic acid, alanine, proline, tyrosine and essential amino acids: methonine and leucine [7].

Traditional processes for the production of Nypa fruticans palm vinegar (NPV) involve a 2-stage fermentation process in which alcohol is initially formed by fermentation process of nipa palm sap with yeast and mold, involving Saccharomyces, Amylomyces, Aspergillus and Candida. The fermentation processes change carbohydrates to alcohols and carbon dioxide, and then, the alcohol is changed to acetic acid by acetic acid bacteria, i.e. Acetobacter aceti. NPV has been previously used as a traditional

\footnotetext{
${ }^{\dagger}$ Presented at the International Conferences on Biomedical Sciences and Medical Technology 2019: June 20 - 21, 2019
} 
medicine because it is believed to have many health benefits. However, there are few studies supporting such assumption. The study of Yusoff et al. [8] reported that NPV could decrease blood glucose levels in mice with induced diabetes mellitus; antioxidant activities were observed in this study.

More empirical studies have identified the immunomodulatory activities of different types of vinegars. Korean persimmon vinegar, for instance, has been confirmed to enhance antibody production [9]. Another study demonstrated that the administration of apple vinegar can potentially improve the expression of immune substances [10]. However, as noted, there are still limited reports on the immunomodulatory effect of NPV. Thus, this study aims to investigate the immunomodulatory effect of NPV from planting areas with different salinities by both in vitro and in vivo analysis.

\section{Materials and methods}

\section{Nypa fruticans palm vinegar (NPV)}

Nypa fruticans palm vinegar (NPV) samples were selected from locally produced NPV from the Kanapnak sub-district, Pak Phanang district, Nakhon Si Thammarat province, Thailand. The samples were collected from 3 different salinity planning areas, including of fresh, brackish and saline. The electrical conductivity $\left(\mathrm{EC} \mathrm{dS} / \mathrm{m}\right.$ at $\left.25^{\circ} \mathrm{C}\right)$ was determined in $1: 5$ soil extract using a conductivity meter and the results were compared according to the soil salinity classification of U.S. Salinity Lab. Staff $[11,12]$.

\section{Animal model}

Male Wistar rats (250 - $300 \mathrm{~g}$ ), which were aged 6 - 8 weeks and obtained from the Nomura Siam International Co., Ltd., were used in this study. All animals were housed and acclimatized in a wellventilated animal transit room (12:12 $\mathrm{h}$ light-dark cycle) at the animal research building, Walailak University, Thailand. Throughout the experiment, the rats were fed food and water by standard methods. The study was authorized for the use of animals in scientific research by the Animal Ethics Committee, Walailak University. The authorization number for the use of animals in scientific research is 006-2017.

\section{Enhancement of the phagocytic activity}

One millilitre of $5 \times 10^{5}$ RAW 264.7 macrophage cells was cultured in a 6-well plate with complete Dulbecco's Modified Eagle Medium (DMEM) (supplemented with $10 \%$ foetal bovine serum, $2 \%$ of $100 \times$ penicillin/streptomycin and $1 \%$ of $100 \times$ amphotericin B). The plate was incubated for $24 \mathrm{~h}$ at $37{ }^{\circ} \mathrm{C}$ in $5 \% \mathrm{CO}_{2}$. Then, $200 \mu \mathrm{lNPV}$ with final concentrations of $100,50,25,12.5,6.25,3.125,1.56$, and 0 $\mu \mathrm{l} / \mathrm{mL}$ was added and incubated for $24 \mathrm{~h}$. Cells were scraped, and rabbit IgG-FITC conjugated latex beads (Cayman, USA) were added directly into pre-warmed cultured medium to a final dilution of 1:400 and incubated at $37{ }^{\circ} \mathrm{C}$ in $5 \% \mathrm{CO}_{2}$ for $1 \mathrm{~h}$. The RAW 264.7 cells were centrifuged at $400 \times \mathrm{g}$ for $5 \mathrm{~min}$. The supernatant was decanted, and the cell pellet was re-suspended in $500 \mu 1$ of assay buffer. The percentage of phagocytic activity was analysed by flow cytometry (BD FACSCalibur ${ }^{\mathrm{TM}}$ ) [13].

\section{Evaluation of the cellular immune response}

Fifty-five rats were used for this study. They were divided into 11 groups of five rats each $(2$ control groups and 9 test groups). The negative control group was fed water, while the positive control group was fed $5 \mathrm{mg} / \mathrm{kg}$ cyclosporine A on day 14 . Test groups were orally administered NPV from different salinity planting areas including fresh, brackish and saline water. NPV was orally administered to rats in 3 different concentrations (1:1, 1:5 and 1:10) with a dosage of $10 \mathrm{~mL} / \mathrm{kg}$ body weight/day for 21 days. Rats were intraperitoneally injected with $0.1 \mathrm{~mL}$ of $20 \%$ sheep red blood cells (SRBC) in sterile normal saline on days 7 and 14. On day 21 , the rats were administered $0.03 \mathrm{~mL}$ of $1 \%$ SRBC into the sub-plantar region of the right hind paw, and at $24 \mathrm{~h}$ after injection, the edema was assessed using a digital plethysmometer [14]. The results were compared between each group. 


\section{Evaluation of the humoral immune response}

Wistar rats were divided into 11 groups and orally administered the treatment at different doses to assess the cellular immune effect. However, rats were treated for 14 days, and the positive control group was orally administered $5 \mathrm{mg} / \mathrm{kg}$ cyclosporine A on day 7 . All rats were subjected to the tail vein blood collection method on day 7 for the determination of the primary antibody response. After blood collection on the same day, $20 \%$ SRBC was peritoneally injected. On day 14, tail vein blood was collected for determining the secondary antibody response. Antibody titres against SRBC were measured by the microtitration technique and calculated as the natural $\log [15]$. The results were compared between each group.

\section{Assessment of the spleen index}

Wistar rats were divided into 11 groups and treated in the same manner for cellular and humoral immune effect assessment. However, the positive control group in this experiment was orally administered $5 \mathrm{mg} / \mathrm{kg}$ cyclosporine A on day 23. At the end of the experiment (day 30), the rats were sacrificed, and the spleens were subjected to weighing. The spleen index was calculated by the following formula for cytotoxicity and immune stimulation assessment [16]: Spleen index $=$ [spleen weight $(\mathrm{mg}) /$ body weight $(\mathrm{g})] \times 100$.

\section{Statistical analysis}

The values are expressed as the mean \pm SEM (standard error of the mean). The results were analysed by 1-way analysis of variance (ANOVA), followed by an independent T-test to determine the statistical significance. The level of significance was set at $p<0.05$.

\section{Results and discussion}

Previous HPLC analysis of the aqueous extract NPV revealed a mixture of organic acids, mainly lactic acid and acetic acid at percentages of 10.35 and $8.68 \%$, respectively [17]. The anti-inflammatory mechanism showed significant lower expression of liver iNOS (inducible nitric oxide synthase) and NFKB transcriptional factor [18]. From previous and recent studies, there is enough evidence that supported NPV as a functional food. Moreover, the significant points of our present study can remove the controversy surrounding functional foods by using an in vivo animal model and in vitro experiments. Many studies have been demonstrating that a functional food supports the concept that food is not only necessary for living but also a source of mental and physical well-being, contributing to the prevention and reduction of risk factors for several diseases or enhancing certain physiological functions. In reference to the immune system, many studies have indicated that not only probiotics but also single micronutrients incorporated into functional foods contribute to an enhancement of immunocompetence. The effective food components that have been demonstrated to have immunomodulatory effects were probiotics [19-21], trace elements [22,23] and dietary antioxidants [24].

\section{Effect on the phagocytic activity}

To test the effect of NPV on the phagocytic activity, which is an important mechanism of innate immunity, NPV from 3 different salinity planting areas was used to directly treat RAW 264.7 macrophage cells, followed by the addition of rabbit IgG-FITC conjugated latex beads. The percentage of active phagocytic cells was determined by flow cytometry. The results showed that the phagocytic activity of macrophage cells that were treated with NPV from the saline planting areas showed significant increases with all of the NPV concentrations when compared to negative untreated cells, while only high concentrations $(25$ and $12.5 \mu \mathrm{l} / \mathrm{mL}$ ) of NPV from the fresh water planting area showed a significant increase in the phagocytic activity. The results of NPV from both saline and fresh water planting areas showed dose-dependent enhancement of the phagocytic activity of macrophage cells. For NPV from brackish water planting areas, only low concentrations $(3.125$ and $1.56 \mu 1 / \mathrm{mL})$ of NPV-treated cells showed significantly enhanced phagocytic activity, which might be due to the different toxic ingredients 
from different NPV sources. However, the results indicated that NPV could enhance the phagocytic activity of macrophages cells, as shown in Figure 1.

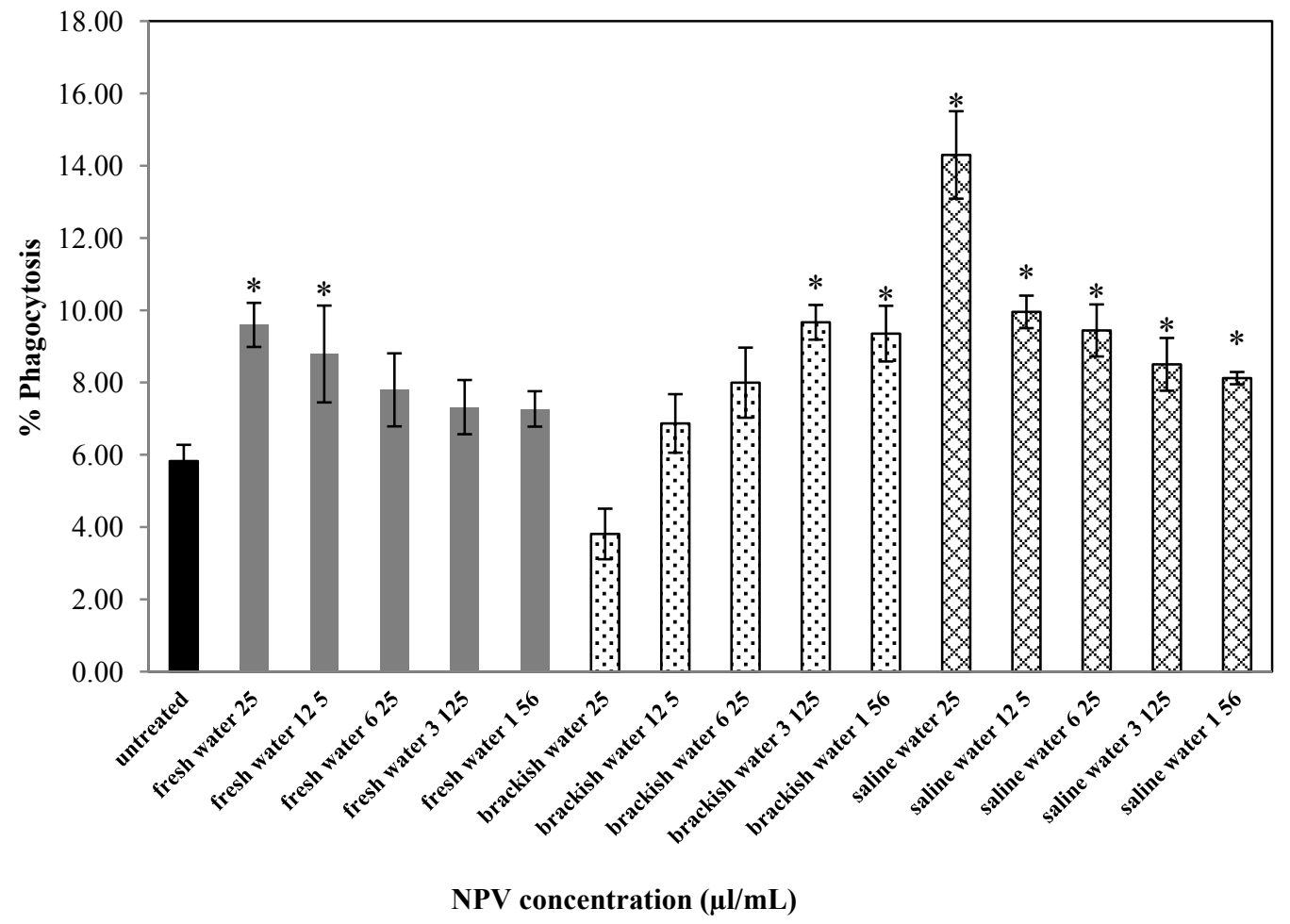

Figure 1 Percentage of phagocytic activity of macrophage cells treated with different concentrations of NPV from 3 different salinity planting areas. The bars show the mean \pm SEM. *; significantly different when compare to the negative control.

Phagocytosis by macrophages is an important nonspecific host-defence (innate immunity) mechanism that occurs immediately after exposure to a foreign antigen. It is also used as an important indicator of the activation of immune function in several studies [25,26]. Our results suggest that NPV can increase the phagocytic potential in macrophages. The phagocytic stimulation activity of NPV has a similar favourable effect to that of most apple cider vinegars (ACVs) that was previously demonstrated by Yagnik and colleagues [27]. The phagocytic activity enhancement might possibly be from the organic acids that are the major constituents of NPV, which is supported by a previous study that demonstrated poultry fed a basal diet supplemented with organic acids had significantly greater phagocytic activity [28].

\section{Cellular immune response modulation by delay-type hypersensitivity inhibition}

To assess the delay-type hypersensitivity (DTH) that indicates the effect on the cellular response, rats were orally administered 3 different concentrations of NPV obtained from 3 different growing areas in parallel with control rats. On day 21 , SRBC was subcutaneously injected into the right hind foot paw, and the edema was evaluated after $24 \mathrm{~h}$. Most of the NPV-treated rats showed significantly lower paw edema when compared with negative untreated rats, except for the rats treated with low doses of NPV from fresh water planting areas, which showed no significant difference. This might be due to the effect 
of different concentrations of active ingredients in NPV from different sources. These modulation effects of NPV were equivalent to cyclosporine A-treated rats, as shown in Figure 2.

Delayed type hypersensitivity (DTH) reaction is a protective localized cell-mediated immuneinflammatory response. It is a mechanism primarily against intracellular pathogens. Upon antigen presentation, T-lymphocytes may become sensitized lymphocytes and will generate a regional abnormal reactive inflammation. This inflammation type is delayed and characterized by cell degeneration and necrosis [29].

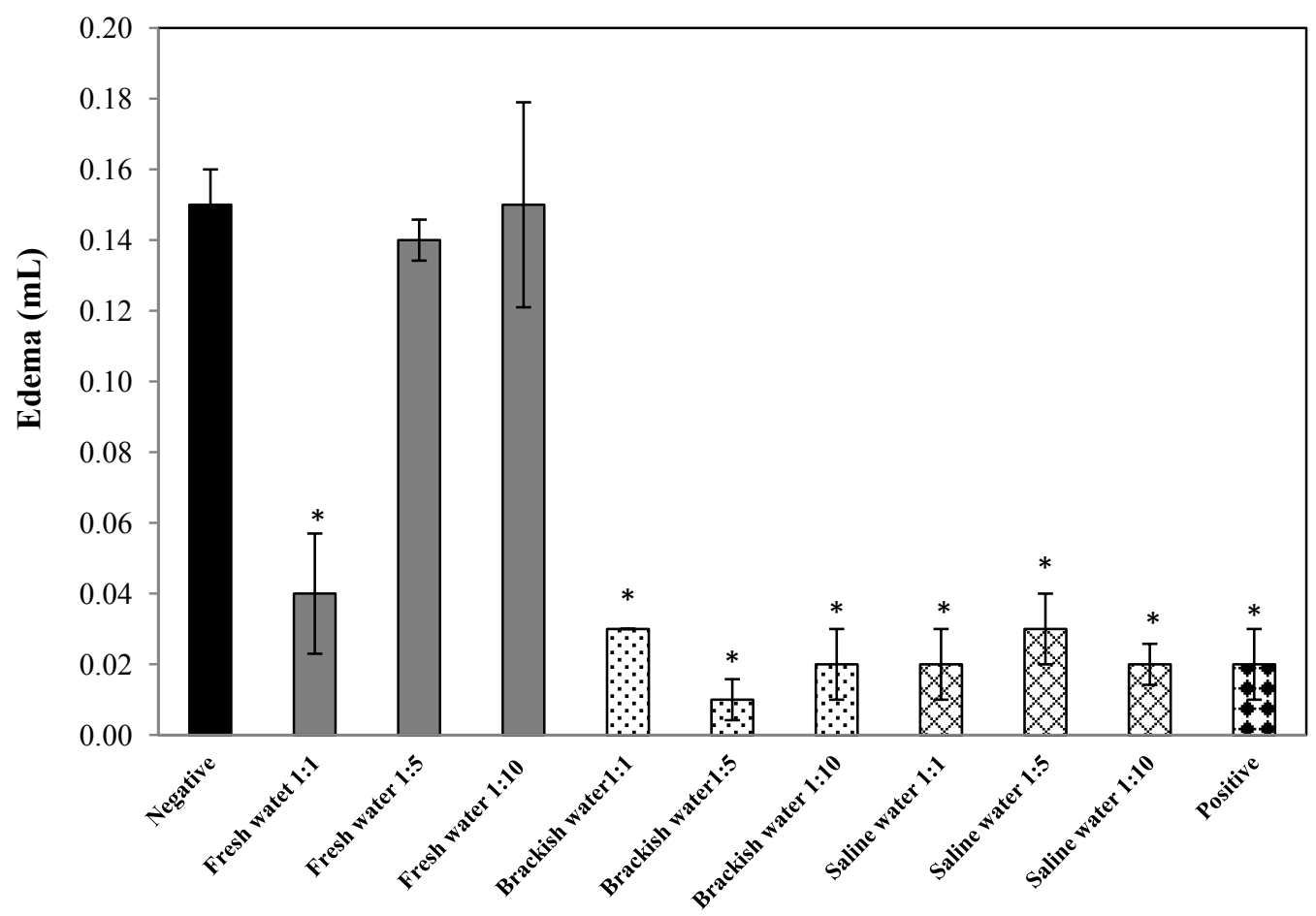

Figure 2 Edema in the right hind paw $24 \mathrm{~h}$ after SRBC injection. The rats were orally administered NPV from different dilutions (1:1, 1:5 and 1:10) and different salinity planting areas (fresh, brackish and sea water). The bars show the mean \pm SEM. ${ }^{*}$; significantly different when compared to the negative control.

The oral administration of NPV in rats showed significantly suppressed hind paw edema compared to the negative control rats. Hind paw edema indicates the strength of the delayed allergic response (DTH), which is mediated by T-cells involved in cellular immunity [30,31]. This inflammation type is delayed and characterized by cell degeneration and necrosis [28]. The inflammation seen in DTH plays a critical protective role against intracellular pathogens. However, such inflammation is often seen against innocuous non-pathogenic antigens leading to severe damage to the tissue, as seen during contact dermatitis against specific allergens. The DTH was considered to be a Th1-driven inflammatory disease; however, treatment with antibodies against IFN- $\gamma$ cytokine showed that such mice still continue to exhibit DTH signs [32]. In addition, the importance of Th17 cells in DTH disease progression was highlighted in IL-17 knockout mice, which showed significantly reduced ear swelling in an allergenic contact dermatitis model [33]. Therefore, it is likely that both Th1 and Th17 cells play an effector role in DTH. A previous study demonstrated that vinegar or acetic acid could suppress the inflammatory response by inhibiting Th1 and Th17 in an animal experiment [34]. Thus, from previous data and our recent results, it is 
suggested that NPV has immunomodulatory activity, which could be related to the inhibition of both the Th1 and Th17 pathways.

\section{Humoral immune response modulation}

To assess the effect of NPV on the humoral immune response in an animal model, rats were orally administered different concentrations and of NPV from different growing areas. The primary and secondary immune responses were evaluated by determining the antibody titre. Blood from the tail veins of rats was collected. It is interesting that low concentrations of NPV (1:10 dilution) showed significantly increased antibody titres for all 3 NPVs from different salinity planting areas when compared to the negative control. Moreover, anti-SRBC from the secondary response of most NPV-treated groups was significantly higher than the primary response, while the response was non-significant in the untreated negative control group. The results are shown in Table 1 and Figure 3. The results clearly suggest that NPV feeding can enhance the antibody response effects.

Table 1 Anti-SRBC titre of rats fed daily with different concentrations of NPV from different salinity planting areas for the primary (day 7) and secondary (day 14) immune response. The data are the mean \pm SEM.

\begin{tabular}{ccc}
\hline \multirow{2}{*}{ Rat groups } & \multicolumn{2}{c}{ Anti-SRBC (mean \pm SEM) } \\
\cline { 2 - 3 } Negative control & Primary response & Secondary response \\
Positive control & $10.05 \pm 3.86$ & $12.88 \pm 1.56$ \\
NPV-Fresh water 1:1 & $20.42 \pm 1.91^{\mathrm{a}}$ & $16.00 \pm 0.00$ \\
NPV-Fresh water 1:5 & $10.48 \pm 0.83$ & $15.31 \pm 2.68$ \\
NPV-Freshwater 1:10 & $13.98 \pm 4.43$ & $19.77 \pm 6.27^{\mathrm{b}}$ \\
\hline NPV-Brackish water 1:1 & $10.76 \pm 5.96$ & $25.75 \pm 3.13^{\mathrm{a}, \mathrm{b}}$ \\
NPV-Brackish water 1:5 & $6.66 \pm 1.60$ & $14.83 \pm 1.17^{\mathrm{b}}$ \\
NPV-Brackish water 1:10 & $7.77 \pm 2.12$ & $18.21 \pm 2.21^{\mathrm{b}}$ \\
\hline NPV-Saline water 1:1 & $12.02 \pm 4.06$ & $21.31 \pm 4.96^{\mathrm{a}, \mathrm{b}}$ \\
NPV-Saline water 1:5 & $14.44 \pm 1.56$ & $18.21 \pm 2.21$ \\
NPV-Saline water 1:10 & $10.48 \pm 0.83$ & $17.66 \pm 1.66^{\mathrm{b}}$ \\
\hline
\end{tabular}

a; significantly different when compared to the negative group, b; significantly different when compared to the primary response. 

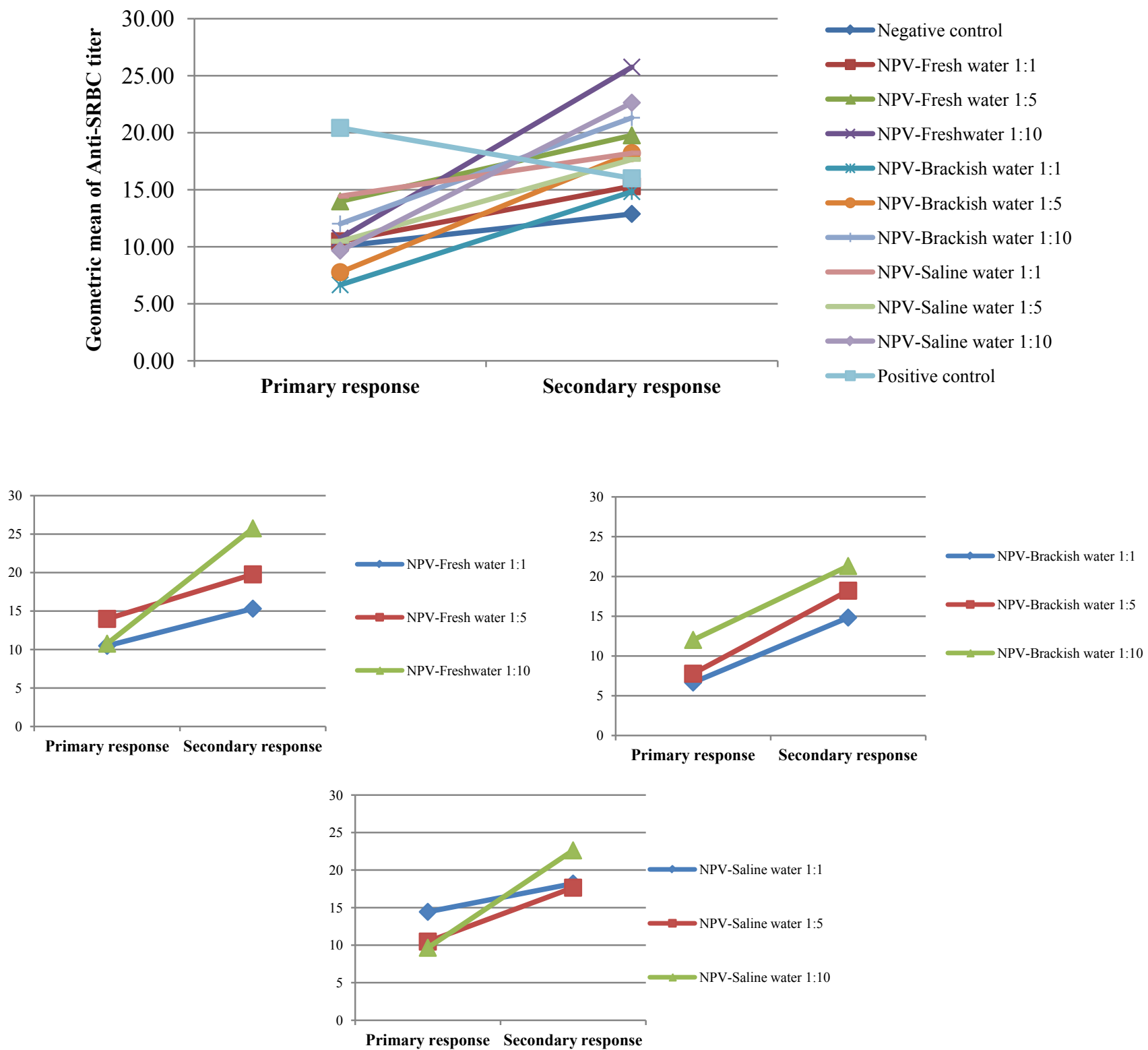

Figure 3 Anti-SRBC titre on the primary (day 7) and secondary (day 14) immune responses of rats that were orally administered different concentrations of NPV from 3 different salinity planting areas on a daily basis.

\section{Spleen index}

Rats were treated with different NPV concentrations from different salinity planting areas for 30 days. Then, the rats were sacrificed, and the spleen was weighed. Both rat groups that were fed with NPV from fresh and brackish water planting areas at the high concentration (1:1 dilution) displayed significant increases in the spleen index when compared to the negative control. This means that the spleens in these 
groups showed an enlarged appearance. However, the medium and low concentrations (1:5 and 1:10) displayed no significant difference in the spleen index when compared to the negative control. In summary, the results indicated that NPV in an appropriate consumption volume did not influence spleen size enlargement. The results are shown in Figure 4.

There are studies that have demonstrated the antibody production enhancement and lymphoid organ effect of vinegar intake in animal models. For example, in evaluating the effects of probiotics and vinegar as dietary supplements on the antibody production of broiler chickens, it was found that chickens had improved immune response against SRBC inoculation, especially in the primary response [35]. The vinegars were known as huge sources of probiotics that can influence microbial communities in the intestine and cease the growth of pathogens [36]. The results of our study were consistent with previous studies since NPV showed the enhancement of specific antibody production; however, there were significant increases, especially in the secondary response. Other evidence that supported vinegar enhancing antibody production is that it is composed of polyphenolics, and a flavonoid was reported to be a component in NPV [8] and play a role in immunoglobulin stimulation or the immunomodulation effect [37]. Moreover, a previous study also determined the effect of probiotics and vinegar on the spleen size; however, there was no effect on the spleen size of animals [36]. The findings of previous studies are sustained by the present study.

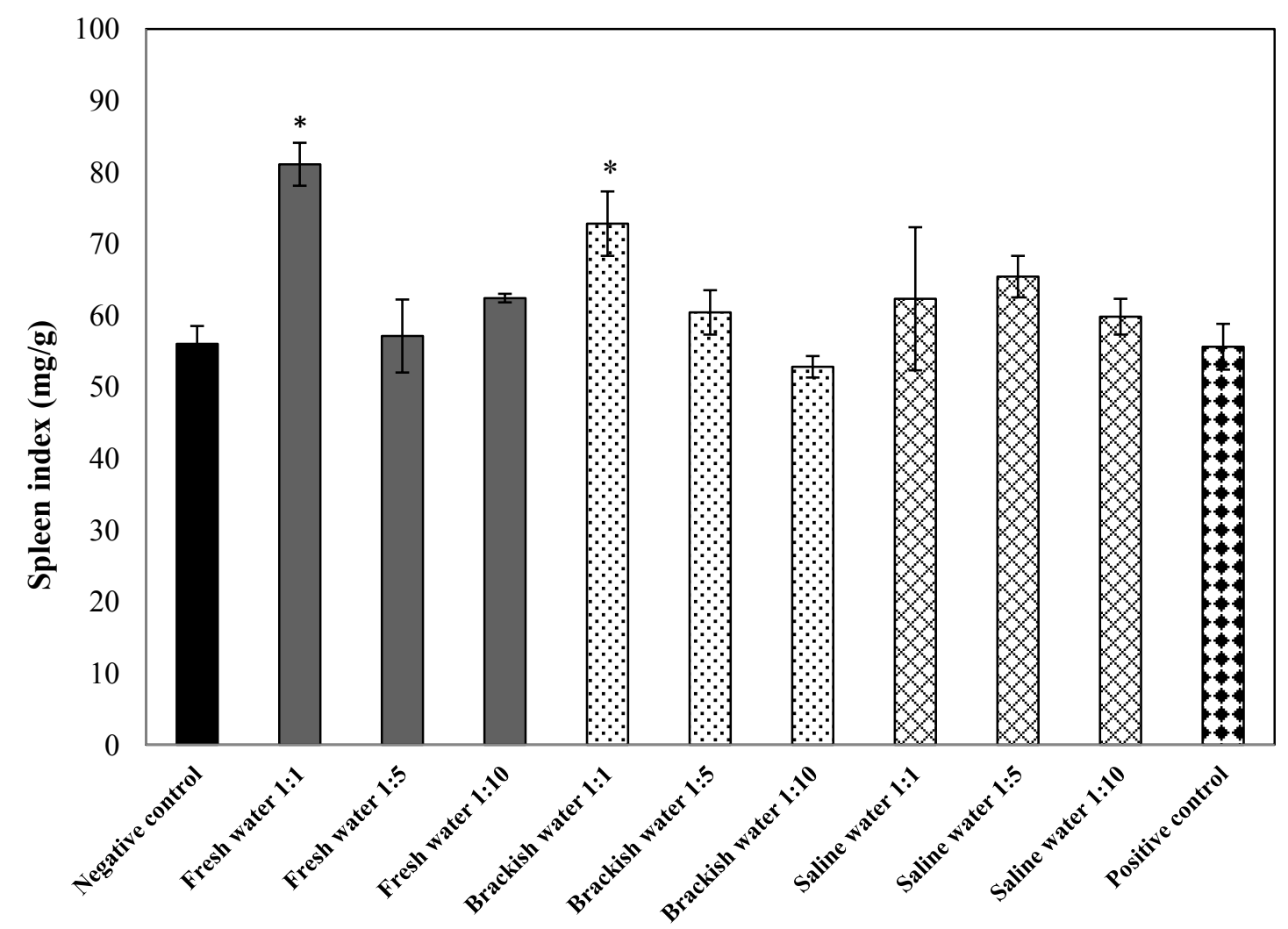

Figure 4 Spleen index of rats that were administered different concentrations of NPV from different salinity planting areas for 30 days. The bars show the mean \pm SEM. *; significantly different when compared to the negative control. 
Especially in the Pak Phanang River Basin in the sub-district of Kanapnak, Pak Phanang district, Naknon Si Thammarat province, the southern east coast of Thailand, the nipa palm is an important economic field crop. Villagers cultivate the nipa palm as their primary source of income in producing various products including sugar, vinegar, whisky, roofing, and cigarette wrap paper. The salinity of planting areas ranges from fresh to brackish and saline water [38]. Salinity has been considered to be an important requirement for the occurrence of Nypa fruticans [39]. It grows well in very low salinity areas (below $5 \mathrm{ppt}$ ), as well as in freshwater outflow [32]. The previous studies showed that high salinity is a limiting factor for the growth of Nypa fruticans. With higher salinity, fewer new leaves are produced in juvenile and mature plants [40]. From these data, the salinity from different planting areas was the possible factor that affected the different active ingredients of NPV. The different active ingredients of NPV might reflect the immunomodulatory effect, as demonstrated in our results. However, the immunomodulatory effects of NPV from different salinity planting areas of southern Thailand showed the same trend, as clearly shown in the results.

\section{Conclusions}

Locally produced NPV form Nakhon Si Thammarat province, Thailand, exhibited enhancement of phagocytic activity in vitro, modulated cellular immune response of delay-type hypersensitivity inhibition (reduced edema) in vivo and modulated humoral immune response by enhanced antibody production in vivo. Moreover, it was affected to spleen with appropriated consumption. However, there was still a limited investigation of the mechanism of the in vitro and in vivo immunomodulatory effect of NPV vinegar with animal testing. Further, mechanistic and cytokine-associated mechanistic investigations are needed to uncover the underlying immunomodulatory activities of different NPV samples. This study concludes that NPV has the ability to modulate either the innate immune response (phagocytic activity) and adaptive immune response (cellular and humoral immunity), and the NPV benefits to human health can be investigated as well.

\section{Acknowledgements}

This research was supported by a grant from the cooperation projects to develop research-based areas between the Thailand Research Fund (TRF) and Walailak University Funds (WU 58611 and $03 / 2561)$.

\section{References}

[1] F Shahidi, J McDonald, A Chandrasekara and Y Zhong. Phytochemicals of foods, beverages and fruit vinegars: Chemistry and health effects. Asia. Pac. J. Clin. Nutr. 2008; 17, 380-2.

[2] NH Budak, E Aykin, AC Seydim, AK Greene and ZB Guzel-Seydim. Functional properties of vinegar. J. Food. Sci. 2014; 79, R757-64,

[3] CS Johnston and CA Gaas. Vinegar: Medicinal uses and antiglycemic effect. Med. Gen. Med. 2006; 8, 61 .

[4] R Shadi, W Robyn, G Richard and EH Cassandra. A review of the hypoglycemic effects of vinegar and its potential benefit in gestational diabetes mellitus (GDM). Obstet. Gynecol. Int. J. 2016; 4, 00096.

[5] FO Javier and Z Scott. Sweet sap from palms, a source of beverages, alcohol, vinegar, syrup, and sugar. Vieraea 2013; 41, 91-113.

[6] T Thanikan. A comparative study of the physicochemical, nutritional characteristics and microbiological contamination of fresh nipa palm (Nypa fruticans) Sap. Burapha Sci. J. 2018; 23, 1301-16.

[7] AA Myint. Some chemical properties and antimicrobial activities of Dani -Ye (Fermented Nypa Sap) obtained by traditional tapping process. Univ. Res. J. 2011; 4, 23-37. 
[8] NA Yusoff, MF Yam, HK Beh, KN Abdul Razak, T Widyawati, R Mahmud, M Ahmad and MZ Asmawi. Antidiabetic and antioxidant activities of Nypa fruticans Wurmb. vinegar sample from Malaysia. Asian. Pac. J. Trop. Med. 2015; 8, 595-605.

[9] MY Lee, H Kim and KS Shin. In vitro and in vivo effects of polysaccharides isolated from Korean persimmon vinegar on intestinal immunity. J. Korean. Soc. Appl. Biol. Chem. 2015; 58, 867-76.

[10] R Safari, SH Hoseinifar, S Nejadmoghadam and M Khalili. Apple cider vinegar boosted immunomodulatory and health promoting effects of Lactobacillus casei in common carp (Cyprinus carpio). Fish. Shellfish. Immunol. 2017; 67, 441-8.

[11] LA Richards. US Salinity Lab. Staff. Diagnosis and Improvement of Saline and Alkali Soil. U. S. Government Printing Office, 1954.

[12] S Bado, BP Forster, AMA Ghanim, J Jankowicz-Cieslak, G Berthold and L Luxiang. Protocol for measuring soil salinity. In: Protocols for Pre-Field Screening of Mutants for Salt Tolerance in Rice, Wheat and Barley. Cham, Springer International Publishing, 2016, p. 13-9.

[13] B Taciak, M Białasek, A Braniewska, Z Sas, P Sawicka, Ł Kiraga, T Rygiel and M Król. Evaluation of phenotypic and functional stability of RAW 264.7 cell line through serial passages. PloS One 2018; 13, e0198943.

[14] BK Beh, NE Mohamad, SK Yeap, KL Lim, WY Ho, HM Yusof, SA Sharifuddin, A Jamaluddin, K Long and NB Alitheen. Polyphenolic profiles and the in vivo antioxidant effect of nipa vinegar on paracetamol induced liver damage. RSC Adv. 2016; 6, 63304-13.

[15] AS Tripathi, V Chitra, NW Sheikh, DS Mohale and AP Dewan. Immunomodulatory activity of the methanol extract of Amorphophallus campanulatus (Araceae) tuber. Trop. J. Pharm. Res. 2010; 9, 451-4.

[16] Z Xia, G Wang, C Wan, T Liu, S Wang, B Wang and R Cheng. Expression of NALP3 in the spleen of mice with portal hypertension. J. Huazhong. Univ. Sci. Technol. Med. Sci. 2010; 30, 170-2.

[17] H Liljeberg and I Björck. Delayed gastric emptying rate may explain improved glycaemia in healthy subjects to a starchy meal with added vinegar. Eur. J. Clin. Nutr. 1998; 52, 368-71.

[18] BK Beh, NE Mohamad, SK Yeap, H Ky, SY Boo, JYH Chua, SW Tan, WY Ho, SA Sharifuddin, K Long and NB Alitheen. Anti-obesity and anti-inflammatory effects of synthetic acetic acid vinegar and Nipa vinegar on high-fat-diet-induced obese mice. Sci. Rep. 2017; 7, 6664.

[19] S Racedo, J Villena, S Salva and S Alvarez. Influence of yogurt consumption on the respiratory immune response. Food. Agric. Immunol. 2009; 20, 231-44.

[20] PR Marteau, M de Vrese, CJ Cellier and J Schrezenmeir. Protection from gastrointestinal disease with the use of probiotics. Nutr. Clin. Pract. 2001; 73, 430s-436s.

[21] MV Kumar, G Mohammad and A Jha. Immunomodulation and anticancer potentials of Yogurt probiotic. Excli. J. 2008; 7, 177-84.

[22] PR Hoffmann and MJ Berry. The influence of selenium on immune responses. Mol. Nutr. Food. Res. 2008; 52, 1273-80.

[23] JC Avery and PR Hoffmann. Selenium, selenoproteins, and immunity. Nutrients 2018; 10, 1203.

[24] S Moriguchi and M Muraga. Vitamin E and immunity. Vitam. Horm. 2000; 59, 305-36.

[25] RE Oberley, AA Kevin, N Traci, K Aswani, CC Erika and MS Jeanne. Surfactant proteins A and D enhance the phagocytosis of Chlamydia into THP-1 cells. Am. J. Physiol. Lung. Cell. Mol. Physiol. 2004; 287, L296-L306.

[26] JN Lee, DY Lee, IH Ji, GE Kim, HN Kim, J Sohn, S Kim and CW Kim. Purification of soluble beta-glucan with immune-enhancing activity from the cell wall of yeast. Biosci. Biotechnol. Biochem. 2001; 65, 837-41.

[27] D Yagnik, V Serafin and AJ Shah. Antimicrobial activity of apple cider vinegar against Escherichia coli, Staphylococcus aureus and Candida albicans; downregulating cytokine and microbial protein expression. Sci. Rep. 2018; 8, 1732.

[28] A Heriazon, JA Yager, W Sears and BA Mallard. Induction of delayed-type hypersensitivity and interferon-gamma to Candida albicans and anti-hen-egg white lysozyme antibody as phenotypic markers of enhanced bovine immune response. Vet. Immunol. Immunopathol. 2009; 129, 93-100. 
[29] XQ Huang, L Deng, G Lu, CH He, PQ Wu, ZW Xie and MA Ashraf. Research on the treatment of Pseudomonas aeruginosa pneumonia in children by macrolide antibiotics. Open Med. (Wars) 2015; 10, 479-82.

[30] M Sarfraz, Y Ashraf and S Ashraf. A review: Prevalence and antimicrobial susceptibility profile of listeria species in milk products. Matrix. Sci. Med. 2017; 1, 3-9.

[31] L Teixeira, B Fonseca, B Barboza and J Viola. The role of interferon- $\gamma$ on immune and allergic responses. Memórias do Instituto Oswaldo Cruz. 2005; 100, 137-44.

[32] T Akkoc, PJA de Koning, B Rückert, I Barlan, M Akdis and CA Akdis. Increased activationinduced cell death of high IFN- $\gamma$-producing TH1 cells as a mechanism of TH2 predominance in atopic diseases. J. Allergy. Clin. Immunol. 2008; 121, 652-8.

[33] S Nakae, Y Komiyama, A Nambu, K Sudo, M Iwase, I Homma, K Sekikawa, M Asano and Y Iwakura. Antigen-specific $\mathrm{T}$ cell sensitization is impaired in IL-17-deficient mice, causing suppression of allergic cellular and humoral responses. Immunity 2002; 17, 375-87.

[34] F Shen, J Feng, X Wang, Z Qi, X Shi, Y An, Q Zhang, C Wang, M Liu, B Liu and L Yu. Vinegar treatment prevents the development of murine experimental colitis via inhibition of inflammation and apoptosis. J. Agric. Food. Chem. 2016; 64, 1111-21.

[35] P Allahdo, J Ghodraty, H Zarghi, Z Saadatfar, H Kermanshahi and MRE Dovom. Effect of probiotic and vinegar on growth performance, meat yields, immune responses, and small intestine morphology of broiler chickens. Ital. J. Anim. Sci. 2018; 17, 675-85.

[36] P Hemarajata and J Versalovic. Effects of probiotics on gut microbiota: Mechanisms of intestinal immunomodulation and neuromodulation. Therap. Adv. Gastroenterol. 2013; 6, 39-51.

[37] B Patwardhan and M Gautam. Botanical immunodrugs: scope and opportunities. Drug. Discov. Today 2005; 10, 495-502.

[38] Walailak University. A Study of the Order of Importance of Problems and People Needs for Research into Development of the Pak Phanang Basin Region. Nipa Palm Group, 2004.

[39] DM Alongi. The Energetics of Mangrove Forests. Australian Institute of Marine Science Townsville, Queensland, Australia, 2009.

[40] R Zakaria, N Aslezaeim and B Sofawi. Effects of water properties and soil texture on the growth of a mangrove palm; Nypa fruticans on carey Island, Malaysia. Pak. J. Bot. 2017; 49, 33-9. 